EESTI NSV TEADUSTE AKADEEMIA TOIMETISED. 27. KOIDE

FOCSIKA * MATEMAATIKA, 1978, NR. 4

ИЗВЕСТИЯ АКАДЕМИИ НАУК ЭСТОНСКОП ССР. ТОМ 27 ФИЗИКА * МАТЕМАТИКА, 1978, № 4

\title{
НЕРАВЕНСТВА ТИПА ЧЕБЫШЕВА ДЛЯ РЕШЕНИЯ E-МОДЕЛЕЙ НЕЛИНЕЙНОГО СТОХАСТИЧЕСКОГО ПРОГРАММИРОВАНИЯ
}

EBU TAMM. TSEBOSEVI TOOPI VORRATUSED MITTELINEAARSE STOHHASTILISE PLANEERIMISE E-MUDELITELE

EBU TAMM. TCHEBYCHEFF INEQUALITIES FOR E-MODELS OF NONLINEAR STOCHASTIC PROGRAMMING

(Представлена Н. Алумяэ)

Рассмотрим две задачи стохастического программирования - задачу без ограничений

$$
\min \left\{E f(x, \eta) \mid x \in R^{n}\right\}
$$

и задачу с ограничениями типа равенств

$$
\min \{E f(x, \eta) \mid E g(x, \eta)=0\},
$$

где $R^{n}-n$-мерное евклидово пространство; $\eta \in R^{r}-$ случайный вектор; $f(x, \eta)$ - функция из $R^{n} \times R^{r}$ в $R^{1} ; g(x, \eta)=\left(g_{1}(x, \eta), \ldots\right.$ $\left.\ldots, g_{m}(x, \eta)\right)$ - функция из $R^{n} \times R^{r}$ в $R^{m} ; E-$ математическое ожидание.

Известно, что во многих случаях задачи (1) и (2) как детерминированные задачи нелинейного программирования или не поддаются решению (распределение вектора $\eta$ неизвестно), или их решение сопряжено с трудностями из-за повторных вычислений $r$-кратных интегралов и их производных. Один из способов преодоления этих трудностей - замена задач (1) и (2) задачами

$$
\min \left\{\frac{1}{k} \sum_{i=1}^{k} f\left(x, \eta_{i}\right) \mid x \in R^{n}\right\}
$$

и

$$
\min \left\{\frac{1}{k} \sum_{i=1}^{k} f\left(x, \eta_{i}\right) \mid \frac{1}{k} \sum_{i=1}^{k} g\left(x, \eta_{i}\right)=0\right\}
$$

соответственно, где $\eta_{1}, \ldots, \eta_{k}-$ независимые реализации случайного вектора $\eta$.

В [1,2] была изучена сходимость случайных величин $E\left[f\left(x_{k}, \eta\right) \mid \eta_{1}, \ldots, \eta_{k}\right]$ к величине $\min _{x \in X} E f(x, \eta)$, где $X$ - либо ограниченное замкнутое множество пространства $R^{n}$ или все пространство $R^{n}$, либо компактное метрическое пространство. В [ $\left.{ }^{1}\right]$ исследовалась также сходимость последовательности $x_{k}, k=1,2, \ldots$, определяемой из условий $\frac{1}{k} \sum_{i=1}^{k} f\left(x_{k}, \eta_{i}\right)=\min _{x \in X} \frac{1}{k} \sum_{i=1}^{k} f\left(x, \eta_{i}\right)$. 
В данной работе сформулируем условия, при которых задачи (3) и (4) имеют решения с некоторой положительной вероятностью, и оценим вероятностные расстояния решений задач (3) и (4) от решений задач (1) и (2). Полученные результаты являются прямыми следствиями из теорем 1 и 3 работы $\left[{ }^{3}\right]$.

Предположим:

1. Сушествует локальное решение $x^{*}$ задачи (1).

2. Линейный оператор $E f^{\prime \prime}{ }_{x x}\left(x^{*}, \eta\right)$ положительно определен, т. е. существует $M_{1}>0$ такое, что $u^{\mathrm{T}} E f^{\prime \prime}{ }_{x x}\left(x^{*}, \eta\right) u \geqslant M_{1}\|u\|^{2}$ для любого $u \in R^{n}$.

3. Функция $f(x, \eta)$ дважды непрерывно дифференцируема по $x$ и $\left\|f^{\prime \prime}{ }_{x x}\left(x^{1}, \eta\right)-f^{\prime \prime}{ }_{x x}\left(x^{2}, \eta\right)\right\| \leqslant K_{1}(\eta)\left\|x^{1}-x^{2}\right\|$ для почти всех $\eta$ и любых $x^{1}, x^{2} \in R^{n}$.

4. $E K_{1}(\eta)$ и $\sigma^{2}\left[K_{1}(\eta)\right]$ конечны.

5. $[E f(x, \eta)]^{\prime}=E f_{x}^{\prime}(x, \eta) \quad$ и $\quad[E f(x, \eta)]^{\prime \prime}=E f^{\prime \prime}{ }_{x x}(x, \eta) \quad$ в точке $x=x^{*}$

6. $E\left\|f^{\prime \prime}{ }_{x x}\left(x^{*}, \eta\right)-E f^{\prime \prime}{ }_{x x}\left(x^{*}, \eta\right)\right\|^{2}$ и $\sigma^{2}\left[f^{\prime} x_{i}\left(x^{*}, \eta\right)\right], i=1,2, \ldots, n$, конечны.

Т е о р е м 1. Пусть выполнены условия $1-6$. Тогда для любых постоянных $\delta_{1}, \delta_{2}$ таких, что $0<\delta_{1}<M_{1}, \delta_{2}>0$, и достаточно больших $k$ существует измеримое мнонество $\$\left(k, \delta_{1}, \delta_{2}\right) \subseteq R^{r} \times \ldots \times R^{r}$ такое,

чTO

1) если $\left(\eta_{1}, \ldots, \eta_{k}\right) \in \mathfrak{S}\left(k, \delta_{1}, \delta_{2}\right)$, то задача (3) имеет локальное решение $x_{k}{ }^{*}\left(\eta_{1}, \ldots, \eta_{k}\right)$;

2) $P\left\{\mathfrak{B}\left(k, \delta_{1}, \delta_{2}\right)\right\} \geqslant p\left(k, \delta_{1}, \delta_{2}\right)$;

3) $P\left\{\left\|x_{k}^{*}\left(\eta_{1}, \ldots, \eta_{k}\right)-x^{*}\right\|<\varepsilon \quad u \quad\left(\eta_{1}, \ldots, \eta_{k}\right) \in \mathfrak{B}\left(k, \delta_{1}, \delta_{2}\right)\right\} \geqslant$

$$
\geqslant p\left(k, \delta_{1}, \delta_{2}\right)-\frac{1}{k \varepsilon^{2}\left(M_{1}-\delta_{1}\right)^{2}} \sum_{i=1}^{n} \sigma^{2}\left[f^{\prime} x_{t}\left(x^{*}, \eta\right)\right],
$$

где

$$
\begin{gathered}
p\left(k, \delta_{1}, \delta_{2}\right)=1-\frac{1}{k}\left[\frac{1}{\delta_{1}^{2}} E\left\|f_{x x}^{\prime \prime}\left(x^{*}, \eta\right)-E f_{x x}^{\prime \prime}\left(x^{*}, \eta\right)\right\|^{2}+\right. \\
\left.+\frac{16\left[E K_{1}(\eta)+\delta_{2}\right]^{2}}{\left(M_{1}-\delta_{1}\right)^{4}} \sum_{i=1}^{n} \sigma^{2}\left[f^{\prime} x_{t}\left(x^{*}, \eta\right)\right]+\frac{1}{\delta_{2}^{2}} \sigma^{2}[K(\eta)]\right]-
\end{gathered}
$$

положитльное число.

Сл ед с т и е 1. Если $k \rightarrow \infty$, то $P\left\{\mathfrak{B}\left(k, \delta_{1}, \delta_{2}\right)\right\} \rightarrow 1 u P\left\{\| x_{k}^{*}\left(\eta_{1}, \ldots\right.\right.$ $\left.\left.\ldots, \eta_{k}\right)-x^{*} \|<\varepsilon \quad u\left(\eta_{1}, \ldots, \eta_{k}\right) \in \mathbb{B}\left(k, \delta_{1}, \delta_{2}\right)\right\} \rightarrow 1$.

Пусть, далее, $x^{*}$ - локальное решение задачи (2). Обозначим лагранжиан задачи (2) через $L(x, \lambda)$, т. $\quad$ е. $L(x, \lambda)=E f(x, \eta)+$ $+\lambda^{\mathrm{T}} E g(x, \eta), \lambda \in R^{m}$. Дополним условия $3-6$ условиями:

7. Существует точка локального минимума $x^{*}$ задачи (2).

8. Функция $g(x, \eta)$ дважды непрерывно дифференцируема по $x$, причем $\left\|g^{\prime \prime}{ }_{x x}\left(x^{1}, \eta\right)-g^{\prime \prime}{ }_{x x}\left(x^{2}, \eta\right)\right\| \leqslant K_{2}(\eta), \quad\left\|g^{\prime \prime}{ }_{x x}(x, \eta)\right\| \leqslant R(\eta)$ для почти всех $\eta \in R^{r}$ и любых $x, x^{1}, x^{2} \in R^{n}$.

9. $E K_{2}(\eta), E R(\eta), \sigma^{2}\left[K_{2}(\eta)\right]$ и $\sigma^{2}[R(\eta)]$ конечны.

10. $[E g(x, \eta)]^{\prime}=E g_{x}^{\prime}(x, \eta)$ и $[E g(x, \eta)]^{\prime \prime}=E g^{\prime \prime}{ }_{x x}(x, \eta) \quad$ в точке $x=x^{*}$.

11. Для некоторого $\mathrm{Q}>0$ верно $\left\|\left[E_{g^{\prime}}\left(x^{*}, \eta\right)\right]^{\mathrm{T}} v\right\| \geqslant \mathrm{Q}\|v\|$ при любом $v \in R^{m}$.

Условия 7 и 11 означают, что существует единственный вектор $\lambda^{*} \in R^{m}$ такой, что $L_{x}^{\prime}\left(x^{*}, \lambda^{*}\right)=0$. 
12. Для некоторого $M_{2}>0$ верно $u^{\mathrm{T}} L^{\prime \prime}{ }_{x x}\left(x^{*}, \lambda^{*}\right) u=u^{\mathrm{T}} E f^{\prime \prime}{ }_{x x}\left(x^{*}, \eta\right) u+$ $+u^{\mathrm{T}} \sum_{j=1}^{m} \lambda_{j}^{*} E g_{j x x}^{\prime \prime}\left(x^{*}, \eta\right) u \geqslant M_{2}\|u\|^{2} \quad$ при любом $u \in R^{n}$.

13. ${ }^{j=1} E\left\|g^{\prime}{ }_{x}\left(x^{*}, \eta\right)-E_{g^{\prime}}\left(x^{*}, \eta\right)\right\|^{2}, \quad E\left\|g^{\prime \prime}{ }_{x x}\left(x^{*}, \eta\right)-E g^{\prime \prime}{ }_{x x}\left(x^{*}, \eta\right)\right\|^{2}$ $\sigma^{2}\left[g_{j}\left(x^{*}, \eta\right)\right], j=1,2, \ldots, m$, конечны.

Т еор ем а 2. Пусть выполнены условия 3-13. Тогда для любых постоянных $\gamma_{1}, \gamma_{2}, \ldots$, $\gamma_{5}$ таких, что $0<\gamma_{1}<M_{2}, 0<\gamma_{2}<\mathrm{e}, \gamma_{3}>0$, $\gamma_{4}>0, \gamma_{5}>0, u$ достаточно больших $k$ существует множество $\mathfrak{B}\left(k, \gamma_{1}, \ldots, \gamma_{5}\right) \subseteq \underbrace{R^{r} \times \ldots \times R^{r}}$ такое, что

1) если $\left(\eta_{1}, \ldots, \eta_{k}\right) \in \mathfrak{W}\left(k, \gamma_{1}, \ldots, \gamma_{5}\right)$, то задача (4) имеет решение $x_{k}{ }^{*}\left(\eta_{1}, \ldots, \eta_{k}\right)$;

2) $P\left\{\mathfrak{W}\left(k, \gamma_{1}, \ldots, \gamma_{5}\right)\right\} \geqslant q\left(k, \gamma_{1}, \ldots, \gamma_{5}\right)$;

3) $P\left\{\left\|x_{R}^{*}\left(\eta_{1}, \ldots, \eta_{k}\right)-x^{*}\right\|<\varepsilon \quad u \quad\left(\eta_{1}, \ldots, \eta_{k}\right) \in \mathfrak{W}\left(k, \gamma_{1}, \ldots, \gamma_{5}\right)\right\} \geqslant$ $\geqslant q\left(k, \gamma_{1}, \ldots, \gamma_{5}\right)-\frac{9\left(D_{3}+\left\|\lambda^{*}\right\| D_{2}+D_{4}\right)}{k \varepsilon^{2}} \quad$ дяя любого $\varepsilon>0$, где

$$
\begin{gathered}
q\left(k, \gamma_{1}, \ldots, \gamma_{5}\right)=1-\frac{1}{k}\left[\frac{D_{1}}{\gamma_{1}^{2}}+\frac{D_{2}}{\gamma_{2}^{2}}+\frac{\sigma^{2}\left[K_{1}(\eta)\right]}{\gamma_{3}^{2}}+\frac{\sigma^{2}\left[K_{2}(\eta)\right]}{\gamma_{4}^{2}}+\right. \\
\left.+\frac{\sigma^{2}[R(\eta)]}{\gamma_{5}^{2}}+144 c_{1}^{4} c_{2}^{2}\left(D_{3}+\left\|\lambda^{*}\right\| D_{2}+D_{4}\right)\right]-
\end{gathered}
$$

положительное число и

$$
\begin{aligned}
& D_{1}=\left(\sqrt{E\left\|f_{x x}^{\prime \prime}\left(x^{*}, \eta\right)-E f_{x x}^{\prime \prime}\left(x^{*}, \eta\right)\right\|^{2}}+\left\|\lambda^{*}\right\| \sqrt{E\left\|g_{x x}^{\prime \prime}\left(x^{*}, \eta\right)-E g_{x x}^{\prime \prime}\left(x^{*}, \eta\right)\right\|^{2}}\right)^{2} \\
& D_{2}=E\left\|g_{x}^{\prime}\left(x^{*}, \eta\right)-E g_{x}^{\prime}\left(x^{*}, \eta\right)\right\|^{2}, \\
& D_{3}=\sum_{i=1}^{n} \sigma^{2}\left[f_{x_{i}}^{\prime}\left(x^{*}, \eta\right)\right] \\
& D_{4}=\sum_{j=1}^{m} \sigma^{2}\left[g_{j}\left(x^{*}, \eta\right)\right]
\end{aligned}
$$

$$
c_{1}=\alpha_{1}+\alpha_{1}^{2} \alpha_{2}^{2} \alpha_{3}+2 \alpha_{1} \alpha_{2} \alpha_{3}+\alpha_{3}
$$$$
\left\|L_{x x}^{\prime \prime}\left(x^{*}, \lambda^{*}\right)\right\|+\gamma_{1}
$$$$
\alpha_{1}=1 /\left(M_{2}-\gamma_{1}\right), \alpha_{2}=\left\|E g^{\prime}{ }_{x}\left(x^{*}, \eta\right)\right\|+\gamma_{2}, \alpha_{3}=\frac{x_{x}}{\left(\varrho-\gamma_{2}\right)^{2}} \text {, }
$$

$$
c_{2}=E K_{1}(\eta)+\left\|\lambda^{*}\right\| E K_{2}(\eta)+3 E R(\eta)+\gamma_{3}+\left\|\lambda^{*}\right\| \gamma_{4}+3 \gamma_{5} .
$$

Следстви е 2. Если $k \rightarrow \infty, \quad$ то $P\left\{\mathfrak{W}\left(k, \gamma_{1}, \ldots, \gamma_{5}\right)\right\} \rightarrow 1 u$ $P\left\{\left\|x_{k}^{*}\left(\eta_{1}, \ldots, \eta_{k}\right)-x^{*}\right\|<s u\left(\eta_{1}, \ldots, \eta_{k}\right) \in \mathfrak{W}\left(k, \gamma_{1}, \ldots, \gamma_{5}\right)\right\} \rightarrow 1$.

\section{Л ИТЕ РА Т У Р А}

1. С ысоев Л. П. Статистические методы обучения, основанные на идентификации учителя. - Автоматика и телемеханика, 1970, № 11, с. 19-28.

2. Фети сов В. Н. Неравенство к методу Монте-Карло. - Теория вероятности и ее применения, 1974 , т. 19 , № 1, с. $224-226$.

3. T a m m, E. Inequalities for the solution of nonlinear programming problems depending on a random parameter. - Math. Operat. u. Statist., (в печати).

Ннститут кибернетики

Академии наук Эстонской ССР
Поступила в редакцию 22/XII 1977 